Génét. Sél. Evol., 1987, 19 (3), 307-320

\title{
Dissémination et voisinage chez une espèce gynodioïque : le cas de Thymus vulgaris (L.)
}

\author{
E. BELHASSEN *, Anne-Charlotte DOCKES *, C. GLIDDON ** \\ et P.H. GOUYON *,*** \\ * C.N.R.S., Centre L. Emberger, BP 5051, F34033 Montpellier Cedex. \\ ** School of Plant Biology, University College of North-Wales, Bangor, LL57 2UW, United Kingdom \\ *** I.N.A.P.G., 16 rue Claude Bernard, F75231 Paris Cedex 05
}

\begin{abstract}
Résumé
Thymus vulgaris est une plante qui colonise de nombreux milieux sans posséder d'adaptation spécifique à la dissémination. L'étude de la dispersion du pollen à l'aide de poudre fluorescente et de la migration des semences par l'action de la pluie et du vent, fournit des résultats cohérents avec les travaux précédents et montre que le thym disperse en moyenne ses gènes sur de faibles distances (inférieures au mètre). Comme pour toutes les mesures de voisinages, les distances de migration sont sous-estimées et l'existence d'une dissémination sur de longues distances est discutée. L'évaluation de la taille des voisinages de 3 populations naturelles de cette espèce gynodioïque révèle une grande variabilité de l'effectif génétique (de 7 à 311 individus). Nous expliquons ces faits par l'existence d'une corrélation positive entre le taux de femelles et la dimension des voisinages.
\end{abstract}

Mots clés : Dissémination, stérilité-mâle, voisinage, Thymus vulgaris.

\author{
Summary \\ Gene dispersal and neighbourhood in a gynodioecious species: \\ the case of Thymus vulgaris $(L)$
}

Thymus vulgaris appears to colonize many habitats without having a specific adaptation for dispersal. We here combine measurements on pollen flow using fluorescent dust, and seed dispersal by rain and wind (which are consistent with previous studies), to estimate an average range of gene flow: gene dispersal in thyme is very limited (under one metre). As with all neighbourhood measures, the computed value underestimates the maximum range of gene flow possible, and the existence of long distance migration is examined. The neighbourhood sizes for 3 populations of this gynodioecious species were found to be variable (from 7 to 311 plants). In fact, it seems that female frequency and neighbourhood size are correlated.

Key words : Dispersal, male-sterility, neighbourhood, Thymus vulgaris. 


\section{Introduction}

La mesure des flux génétiques est une étape cruciale vers la compréhension de l'évolution des systèmes génétiques des êtres vivants. Dans le modèle « isolation by distance » de WRIGHT $(1943,1946)$, la population n'est plus considérée comme une unité panmictique. Par contre le voisinage, qui constitue une échelle plus fine, y est défini comme la surface dans laquelle se trouvent les parents d'un individu situé au centre. WRIGHT prévoit l'apparition d'une différenciationn génétique dans les populations quand l'effectif génétique (CROw \& KimURA, 1970 d'après WRIGHT, 1931) est inférieur à 200 individus. Les mesures de voisinages qui ont été faites depuis, sur des espèces animales, montrent que les effectifs génétiques sont en général supérieurs à 200 individus (Kerster, 1964 ; Greenwood, 1975 ; Barrowclough, 1980 ; Begon et al., 1980), ce qui suggère une faible structuration génétique des populations. Chez les végétaux, la dispersion des gènes (effectuée à la fois par le pollen et par les graines) peut être très faible, ce qui peut conduire à des effets de dérive génétique importants à l'intérieur des populations.

Chez le thym, Brabant et al., (1980) ont établi, en observant le comportement des pollinisateurs et grâce à un modèle prédisant le taux d'autofécondation des individus en fonction de leur isolement, que la distance de migration du pollen est comprise entre 0,50 et $1 \mathrm{~m}$, mais il s'agit d'estimations très indirectes. D'autre part, la dispersion des graines de thym est connue comme très limitée ; les schizocarpes des Labiées ne sont jamais cités comme de bons exemples d'adaptation à la dissémination. Les expériences faites sur ce sujet (GouYon et al., 1987) ne permettent pas de définir une distance moyenne de migration des graines.

Les estimations de taille de voisinage (Kerster \& LeVIN, 1968 ; LeVIN \& Kerster, 1968, 1969 ; Schaal \& Levin, 1978 ; Beattie \& Culver, 1979 ; SchaAl, 1980 ; Schmitt, 1980 ; Cahalan \& Gliddon, 1985) concernent en général des espèces hermaphrodites. En étudiant une espèce gynodiö̈que comme Thymus vulgaris (il existe à la fois des individus hermaphrodites et des individus femelles), nous cherchons à savoir si la proportion de femelles a un impact sur la dimension du voisinage. Nous espérons ainsi apporter un élément de compréhension supplémentaire à l'analyse de ce polymorphisme sexuel.

\section{Matériel et méthodes}

\section{A. Dispersion du pollen}

L'utilisation de la poudre fluorescente comme traceur de la migration du pollen (Stockhouse, 1976 ; WASER \& Price, 1982 ; Olivieri et al., 1983) permet d'obtenir des estimations plus précises que l'observation directe. En effet, l'observation des pollinisateurs n'est réalisable que pendant une durée limitée et pour des pollinisateurs suffisamment visibles. La mesure de dispersion de la poudre fluorescente prend en compte l'activité intégrale de tous les pollinisateurs. Cette poudre, qui n'est pas particulièrement lumineuse à la lumière du jour, émet une forte fluorescence lorsqu'elle est éclairée par des ultra-violets. Chaque grain de poudre peut être alors repéré de nuit grâce à une lampe portative à ultra-violets. 
Dans le cas du thym, une approche particulière est possible : les hermaphrodites ont été considérés uniquement comme sources du pollen, les femelles comme cibles de la migration pollinique.

Nous avons étudié 2 stations, l'une dans la garrigue (station LJ2), l'autre près de notre laboratoire (station CP). Dans la station LJ2 (qui a été antérieurement étudiée par Bonnemaison, 1980), la densité est faible (4,7 individus par $\mathrm{m}^{2}$ ) et femelles et hermaphrodites ne sont pas éloignés les uns des autres (figure 1a). Deux hermaphrodites, distants respectivement de 0,50 et $9 \mathrm{~m}$ des femelles, ont été marqués. Cette station correspond à un stade de recolonisation de 7 ans après un incendie.

La station CP présente un intérêt particulier car 13 femelles forment une « tache » compacte, et l'hermaphrodite le plus proche en est distant approximativement de $9 \mathrm{~m}$; de plus, il est isolé par une végétation dense. D'autre part, 4 femelles éparses sont situées à un mètre de cet hermaphrodite, mais elles sont cachées par la même végétation dense. Le $2^{\mathrm{e}}$ hermaphrodite le plus proche est situé à une quarantaine de mètres de la tache de femelles (figure $1 \mathrm{~b}$ ). Cette station correspond à un stade de recolonisation très précoce.

La poudre fluorescente a été déposée la nuit, sous éclairage ultra-violet, sur les fleurs de 2 hermaphrodites pour chaque station (une couleur différente est utilisée pour chacun des 2 hermaphrodites). Les nuits suivantes, les femelles dont les styles des fleurs ont été colorés par la foudre fluorescente sont cartographiées, ce qui permet d'estimer la distance moyenne et la variance de dispersion du pollen de la station.

\section{B. Dispersion des semences}

Les 2 facteurs de dissémination que nous avons étudiés sont la pluie et le vent. Cinq femelles et 5 hermaphrodites ont été soumis à l'expérimentation. Les pieds ont été placés à l'extérieur (à l'abri du vent) sur un plan horizontal, afin que les précipitations naturelles d'automne provoquent la chute des diaspores. Pour simuler les irrégularités du sol, un grillage en plastique à mailles serrées recouvre le plan horizontal. Sous chacun des 10 pieds, un quadrillage centimétré permet de mesurer la distance entre les diaspores tombées et la plante-mère. Les individus sont restés 4 jours sous des pluies parfois violentes.

Pour la dissémination par le vent, nous avons placé au laboratoire les 10 individus devant un ventilateur, face à un couloir canalisant les diaspores dispersées. Le même grillage à mailles serrées tapisse le plancher du couloir, afin d'éviter le glissement des diaspores une fois tombées. Le ventilateur produit un vent de $5 \mathrm{~m}$ par seconde, ce qui correspond à la vitesse moyenne du vent en été dans la garrigue.

Les distances parcourues par les graines et les fruits sont mesurées ; ces derniers sont récoltés, afin de déterminer s'ils contiennent des graines et donc s'ils peuvent être considérés comme de véritables diaspores. Deux plantes, l'une femelle et l'autre hermaphrodite, sont conservées à l'abri de la pluie et du vent, et servent de témoins.

\section{Estimation du voisinage}

La surface du voisinage $(\mathrm{Na})$ peut être calculée à partir de la formule WRIGHT (1946) : $\mathrm{Na}=4 \pi \sigma^{2}$ où $\sigma^{2}$ est la variance de dispersion. 
$1 \mathrm{a}$

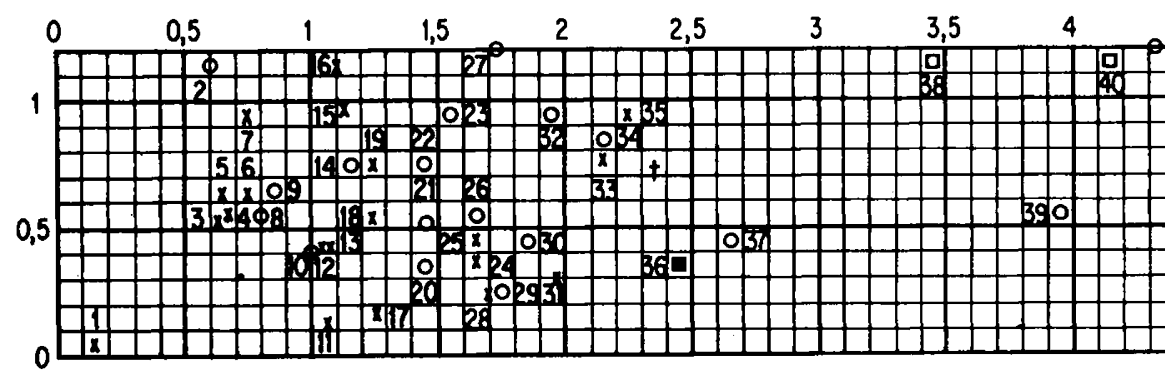

$1 b$

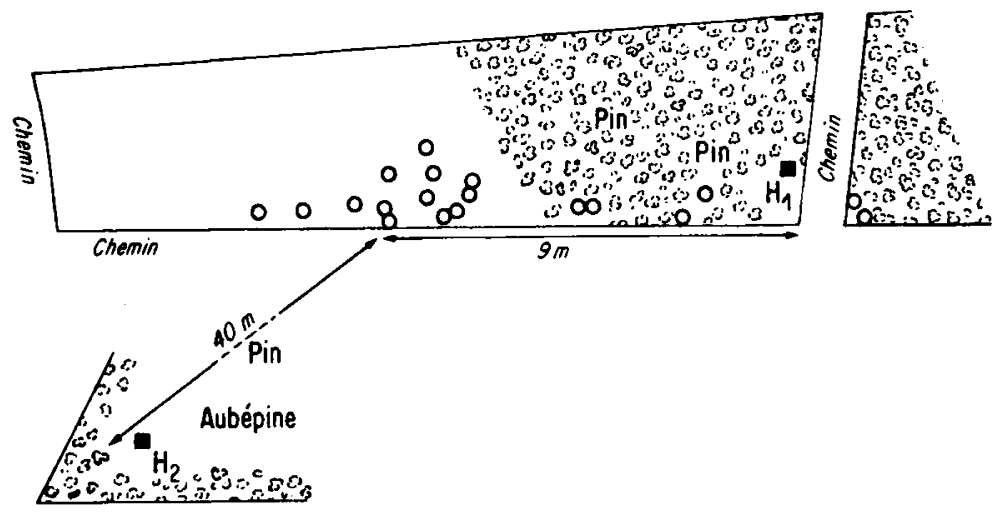

Pour déterminer $\sigma^{2}$ chez un végétal, nous avons utilisé la formule établie dans GLIDDON et al. (1987), qui est applicable aussi bien pour les espèces hermaphrodites que dioïques et gynodioïques :

$$
\sigma^{2}=\sigma_{v}^{2}+\frac{1}{2} t \sigma_{p}^{2}+R h . \sigma_{s h}^{2}+(1-R h) \sigma_{s f}^{2}
$$

avec $\sigma_{\mathrm{v}}{ }^{2}$ : variance de dispersion due à la croissance végétative

$\mathrm{t} \quad$ : proportion d'ovules allofécondés

$\sigma_{\mathrm{p}}^{2}$ : variance de dispersion du pollen

$\mathrm{Rh}$ : proportion de graines issues d'hermaphrodites

$\sigma_{\mathrm{sh}^{2}}{ }^{2}:$ variance de dispersion des semences des hermaphrodites

$\sigma_{\mathrm{st}}{ }^{2}$ : variance de dispersion des semences des femelles.

Les variances axiales de dispersion ont été calculées à partir des expérimentations précédentes, selon la méthode développée en détail par CRAWFORD (1984a).

Dans le cas d'espèces gynodiö̈ques, la signification du paramètre $t$ est plus complexe que dans le cas d'espèces hermaphrodites. La proportion d'ovules allofécondés dépend du taux de graines allofécondées issues des plantes hermaphrodites (soit th. $\mathrm{Rh}$, où th est le taux d'allofécondation mesuré sur les hermaphrodites) et de la proportion de graines issues de femelles qui sont nécessairement allofécondées (soit $1-\mathrm{Rh}$ ). Ainsi : $\mathrm{t}=(1-\mathrm{Rh}+\mathrm{th} \cdot \mathrm{Rh})$. 
Les valeurs de $\mathrm{Na}$ et $\mathrm{Ne}$ (et celles de $\mathrm{Nac}$ et $\mathrm{Nec}$ ) ont été calculées pour 3 populations (tableau 3), dont les paramètres th, h et $\mathrm{d}$ sont connus (VALDEYRon et al., 1977 ; Bonnemaison, 1980). Les valeurs des avantages femelles (x) n'étant pas connues pour les populations, nous avons fixé $x=2$, ce qui correspond à la valeur moyenne de $\mathrm{x}$ (Couvet et al., 1985b). De même, aucune estimation des dépressions de consanguinité n'étant disponible, nous avons considéré ce paramètre comme nul.

\section{Résultats}

La distance de migration moyenne du pollen pour la station $\mathrm{LJ} 2$ est égale à $0,9 \mathrm{~m} \pm 0,17$ (intervalle de confiance à 0,05 ). Pour la station $\mathrm{CP}$, nous n'avons observé aucun transfert de poudre fluorescente entre les plantes en place. Afin de vérifier s'il n'y a effectivement pas eu transport de pollen, nous avons prélevé, en fin de maturation, les fruits des femelles situés dans l'entourage des hermaphrodites marqués. Nous avons trouvé très peu de fruits sur toutes ces plantes ( 200 fruits au total) car une abscission précoce a eu lieu, ce qui confirme la quasi-absence de pollinisation. D'autre part, sur les 200 fruits récoltés, 4 seulement contenaient des graines.

Les résultats concernant la dissémination des diaspores sous l'action de la pluie (figures 2a) montrent que les graines seules migrent en plus grandes proportions que les fruits (en comparaison avec les graines et les fruits non migrants, $\chi^{2}=62$, ddl $=1$, $P<0,001$ ). L'analyse de l'action de la pluie (tableau 1) montre une différence significative entre les distances moyennes de dispersion des graines et des fruits des femelles (respectivement 4,3 et $2,6 \mathrm{~cm}$; test $\mathrm{t}$, ddl $=500, \mathrm{P}<0,001)$; pour les hermaphrodites, la différence est moins nette (respectivement 3,5 et $2,8 \mathrm{~cm}$, ddl $=407, P=0,06$ ). D'autre part, les graines des femelles sont dispersées plus loin que celles des hermaphrodites $(\mathrm{ddl}=522, \mathrm{P}=0,05)$, mais ceci n'est pas vérifié pour les fruits.

La dissémination par le vent (figure 2b) montre qu'il n'y pas de différence significative entre femelles et hermaphodites dans la dispersion des graines $(0,82$ contre $0,83 \mathrm{~m})$ ou des fruits $(1,08$ contre 1,02 ; tableau 1). En outre, les distances moyennes de migration des graines et des fruits sont significativement différentes (respectivement 0,82 et $1,05 \mathrm{~m}$; test $\mathrm{t}$, ddl $=625, \mathrm{P}<0,001$ ). Cependant, les graines dans les fruits sont plus disséminées par les femelles que par les hermaphrodites (en comparaison avec les graines des femelles et des hermaphrodites qui n'ont pas migré, $\chi^{2}=24$, ddl $=1$, $\mathbf{P}<0,001$ ). L'analyse du nombre de graines par fruit (tableau 2) montre que les fruits dispersés par le vent provenant des femelles contiennent significativement plus de graines que les fruits restés en place sur ces femelles (test $t$, ddl $=8, P<0,05$ ). Pour les hermaphrodites, la proportion de fruits pleins qui ont migré n'est pas significativement différente de celle des fruits restés sur la plante témoin (tableau 2).

Ces dernières constatations nous obligent à considérer séparément les femelles et les hermaphrodites lors des calculs des distances moyennes de dispersion des semences. Le calcul de la distance moyenne de dispersion des graines est effectué en pondérant les moyennes obtenues par les proportions respectives de graines et de fruits dispersés dans chacune des 2 expérimentations.

Pour les plantes témoins protégées de la pluie et du vent, une très faible proportion de diaspores (1,5 p. 100 de la totalité des graines disséminées lors des expérimentations) est tombée des 2 plantes-mères, durant la période de dissémination naturelle dans la garrigue (de juin à août). 
Figure 20

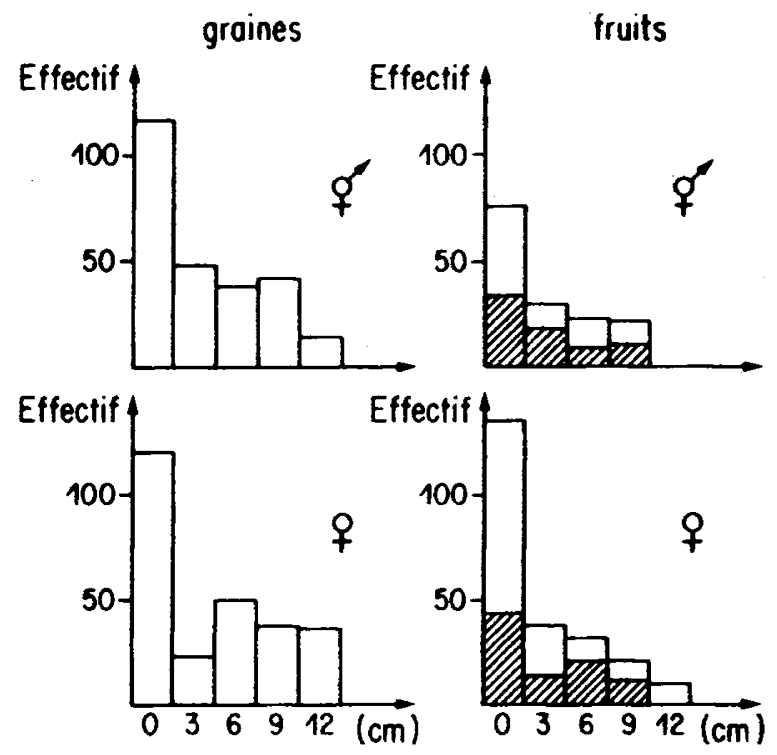

Figure $2 b$

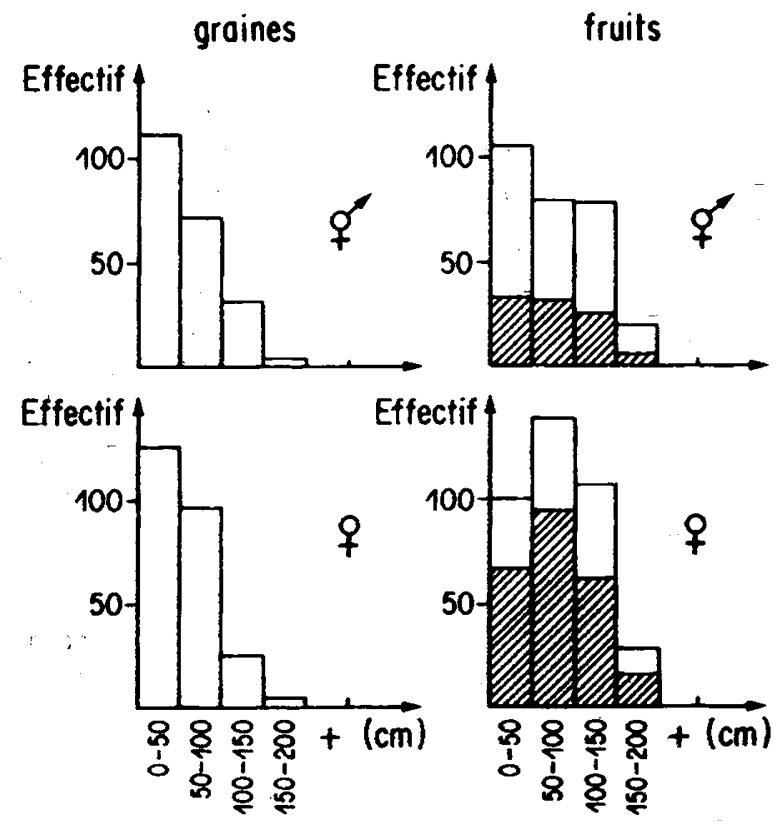

Fig. 2

Histogrammes de dispersion sous l'action de la pluie (a) et du vent (b) pour les graines et les fruits et pour les 2 sexes. Les zones hachurées correspondent aux proportions de fruits pleins.

Histograms of dispersion due to rain (a) and wind (b) for seeds and fruits, and for the 2 sexes. The hatched areas correspond to the proportions of full fruits. 


\section{TABLEAU 1}

Distances moyennes de dispersion exprimées en centimètres (et leur intervalle de confiance à 0,05) pour les 2 sexes, sous l'action de la pluie et du vent et signification statistique par le test $t$ (dans chacune des colonnes, les moyennes suivies de lettres différentes sont significativement différentes 2 à 2 au seuil 0,05).

Mean distances of dispersal expressed in centimetres (and their confidence interval at the 0.05 level) for the 2 sexes, due to rain and wind, and statistical significance judged by t-test (in each column, means followed by different letters differ significantly together at the 0.05 level).

\begin{tabular}{lc|c|c}
\hline \hline & & Pluie & Vent \\
\hline \multirow{3}{*}{ Hermaphrodites } & Graines & $\begin{array}{c}3,5^{\mathrm{a}} \\
( \pm 0,48)\end{array}$ & $\begin{array}{c}83^{\mathrm{a}} \\
( \pm 5,0)\end{array}$ \\
\cline { 3 - 4 } & \multirow{3}{*}{ Fruits } & $\begin{array}{c}2,8^{\mathrm{a}} \\
( \pm 0,53)\end{array}$ & $\begin{array}{c}102^{\mathrm{b}} \\
( \pm 5,6)\end{array}$ \\
\cline { 3 - 4 } & \multirow{3}{*}{ Femelles } & $\begin{array}{c}4,3^{\mathrm{b}} \\
( \pm 0,54)\end{array}$ & $\begin{array}{c}82^{\mathrm{a}} \\
( \pm 4,6)\end{array}$ \\
& \multirow{2}{*}{ Fruines } & $\begin{array}{c}2,6^{\mathrm{a}} \\
( \pm 0,46)\end{array}$ & $( \pm 4,7)$ \\
\cline { 3 - 4 } & & & \\
\hline
\end{tabular}

TABLEAU 2

Comparaison des nombres moyens de graines par fruit (et de leur intervalle de confiance à 0,05) entre des fruits restés en place sur les 10 plantes mères (témoins),

et ceux qui ont migré sous l'action du vent et de la pluie (test $t$ intra-ligne; ${ }^{*}: P<0,05$ ).

Comparison of mean numbers of seeds per fruit (and their confidence interval at 0.05) between fruits remaining on the 10 maternal plants (controls) and those which have migrated due to wind and rain ( $t$ test within line; $*=P<0.05)$.

\begin{tabular}{l|c|c|c|c}
\hline & & Témoins & Pluie & Vent \\
\hline Femelles $(\mathrm{n}=5)$ & $\ldots \ldots \ldots \ldots \ldots$ & $0,78 \pm 0,26$ & $0,73 \pm 0,39$ & $1,15^{*} \pm 0,26$ \\
\hline Hermaphr. $(\mathrm{n}=5)$ & $\ldots \ldots \ldots \ldots \ldots$ & $0,92 \pm 0,34$ & $0,80 \pm 0,32$ & $0,71 \pm 0,40$ \\
\hline \hline
\end{tabular}

Les variances de dispersion nécessaires à la mesure des voisinages ont été calculées sous l'hypothèse d'une distribution de dispersion normale et de moyenne nulle (CRAWFORD, 1984a). Les variances axiales obtenues sont les suivantes :

$$
\begin{aligned}
& \sigma_{\mathrm{p}}^{2}=0,41 \\
& \sigma_{\mathrm{sh}}{ }^{2}=0,23 \\
& \sigma_{\mathrm{sf}^{2}}=0,33
\end{aligned}
$$

Les valeurs de la taille et de l'effectif des voisinages des 3 populations naturelles sont données dans le tableau 3. Les estimations de $\mathrm{Na}$ et de $\mathrm{Ne}$ sont relativement fiables, malgré le fait que $\sigma_{\mathrm{p}}^{2}$ n'a été mesuré que pour une densité unique et qu'il peut diminuer quand la densité augmente. En effet, les valeurs de Nec correspondant à $\sigma_{\mathrm{p}}{ }^{2}=0$, nous voyons que les mêmes ordres de grandeur sont trouvés (tableau 3). 


\section{TABLEAU 3}

Effectifs (Ne) et surfaces $(\mathrm{Na})$ des voisinages moyens de 3 populations du Languedoc;

$h$ est la proportion d'hermaphrodites, th, le taux d'allofécondation des hermaphrodites et $d$ la densité en individus fleuris.

Les valeurs de Nac et Nec ("voisinages cytoplasmiques") sont mises entre parenthèses. Les variances axiales de dispersion sont égales à 0,41 pour le pollen, 0,33 pour les semences de femelles, et 0,23 pour celles des hermaphrodites.

Average effective sizes ( $\mathrm{Ne}$ ) and areas ( $\mathrm{Na}$ ) of neighbourhoods for 3 populations of Languedoc (France); $h$ is the proportion of hermaphrodites, th the rate of cross-fertilisation in hermaphrodites and $d$ the density of flowering plants.

The values of Nac and Nec ("cytoplasmic neighbourhoods") are given in parentheses.

The axial variances of dispersal are 0.41 for the pollen, 0.33 for seeds from females and 0.23 for seeds from hermaphrodites.

\begin{tabular}{|c|c|c|c|c|c|c|}
\hline & $\mathrm{h}$ & th & $\underset{\text { (Ind./m²) }}{\mathrm{d}}$ & $\begin{array}{c}\mathrm{Na} \\
\left(\mathrm{m}^{2}\right)\end{array}$ & $\begin{array}{l}\mathrm{Ne} \\
\text { (Ind.) }\end{array}$ & $\begin{array}{c}\text { Sources } \\
\text { des paramètres }\end{array}$ \\
\hline Les Chênes & 0,66 & 0,64 & 1,3 & $\begin{array}{c}5,8 \\
(3,6)\end{array}$ & $\begin{array}{c}7 \\
(5)\end{array}$ & $\begin{array}{l}\text { VALDEYRON } \\
\text { et al. (1977) }\end{array}$ \\
\hline Le Vigan & 0,46 & 0,90 & 2,1 & $\begin{array}{c}6,4 \\
(3,9)\end{array}$ & $\begin{array}{l}13 \\
(8)\end{array}$ & $\begin{array}{l}\text { VALDEYRON } \\
\text { et al. (1977) }\end{array}$ \\
\hline La Jasse 2 & 0,27 & 0,71 & 48,7 & $\begin{array}{c}6,5 \\
(4,1)\end{array}$ & $\begin{array}{c}311 \\
(198)\end{array}$ & $\begin{array}{l}\text { COUVET } \\
\text { et al. }(1986)\end{array}$ \\
\hline
\end{tabular}

\section{Discussion}

\section{A. Dissémination du pollen}

La valeur de la distance moyenne de migration du pollen $(0,9 \mathrm{~m} \pm 0,17)$ est cohérente avec les résultats de BRABANT et al., (1980) puisqu'elle est comprise dans l'intervalle 0,50 à $1 \mathrm{~m}$, estimé à partir de l'observation des pollinisateurs. Dans la station $\mathrm{CP}$, les femelles cachées par la végétation dense, mais proches du premier hermaphrodite, n'ont pas été pollinisées, ce qui confirme la conclusion de BRABANT et al. (1980) : la visibilité des plantes en fleurs est un facteur important de la pollinisation. Dans la station CP, il existe une faible pollinisation ( 2 p. 100 de fruits portent des graines) que nous n'avons pas détectée avec la poudre fluorescente ; ceci peut s'expliquer par le fait que la durée de marquage des hermaphrodites a été inférieure à la durée totale de floraison. Il peut donc exister des transferts de pollen sur des distances bien supérieures (jusqu'à $9 \mathrm{~m}$ ) à la distance de dispersion moyenne de la station LJ2.

Il faut remarquer que la méthode utilisant la poudre fluorescente ne permet pas d'évaluer les possibilités de transferts de pollen, puisque la présence de poudre fluorescente sur un stigmate de fleur femelle indique une dissémination et non pas une pollinisation réelle. De plus, le nombre de grains de poudre fluorescente n'ayant pas été quantifié, il peut exister un biais dans la mesure de la distance moyenne de migration du pollen (ainsi que dans celle de la variance). En effet, les femelles les plus proches de l'hermaphrodite marqué devraient recevoir une plus grande quantité de poudre fluorescente (ou de pollen) que celles qui en sont plus éloignées. La pollinisation efficace résultant de la compétition entre les grains de pollen des différents hermaphrodites, est 
dépendante des quantités des différents pollens présentes sur un stigmate. De ce fait, pour estimer de façon exacte la distance moyenne de dispersion du pollen, il serait nécessaire de pondérer les distances de migrations observées par la quantité de poudre fluorescente reçue. Ainsi, la distance moyenne que nous avons obtenue est probablement surestimée.

Si la technique utilisant la poudre fluorescente conduit à une surestimation de la dispersion du pollen, il faut remarquer que l'observation directe des pollinisateurs aboutit à une sous-estimation. En effet, cette méthode suppose le plus souvent que le pollen collecté, est déposé uniquement sur la prochaine plante visitée (KERSTER \& LEVIN, 1968 ; Levin \& Kerster, 1969 ; Levin et al., 1971; SchaAl, 1980). Il a été montré que les pollinisateurs peuvent transporter le pollen au-delà de la $1^{\text {re }}$ plante visitée (Schmitt, 1980 ; Thomson \& Plowright, 1980). Ainsi, l'observation simple des pollinisateurs conduit à une sous-estimation des transferts de pollen. WASER \& PRICE (1982) ont montré que les transferts de poudre fluorescente (le pollinisateur étant un colibri) sont statistiquement similaires qualitativement et quantitativement à ceux du pollen. Il semble donc que la méthode utilisant la poudre fluorescente permet d'obtenir des estimations correctes des transferts de pollen entre plantes.

\section{B. Dissémination des semences}

Les expériences précédentes montrent que le vent et la pluie sont des facteurs indispensables pour la dispersion des semences ; en effet, très peu de diaspores sont tombées des plantes témoins $(1,5$ p. 100). De plus, les fruits, que l'on peut considérer comme de véritables diaspores, permettent la dissémination d'une fraction importante de graines (45 p. 100 de l'ensemble des graines disséminées), et ces mêmes fruits sont transportés plus loin que les graines sous l'action du vent. Les graines de thym migrent légèrement plus loin que les fruits sous l'action de la pluie; ceci peut être dû au ruissellement de l'eau qui entraîne plus facilement les graines (à cause de leur faible taille $-0,5 \mathrm{~mm}$ en moyenne).

Les différences dans les proportions de fruits pleins disséminés entre femelles et hermaphrodites sont d'autant plus difficilement interprétables, que ces différences sont observées uniquement sous l'action du vent. Cependant, ce résultat semble fiable car chacune des 5 femelles a présenté la même tendance à disperser des fruits plus chargés en graines (tableau 2). Deux explications sont possibles : ou bien les fruits pleins des femelles s'envolent plus facilement sous l'action du vent par rapport aux hermaphrodites, ou bien les fruits vides restent mieux accrochés à la plante mère quand elle est femelle. Les répercussions de ce phénomène sur la valeur des distances moyennes de dispersion des graines $(\mathrm{Dg})$ sont faibles : $\mathrm{Dg}$ vaut $\mathbf{0 , 4} \mathrm{m}$ pour les hermaphrodites, et $0,6 \mathrm{~m}$ pour les femelles. Mais la pondération de ces distances par les fréquences relatives des hermaphrodites et des femelles pourra accentuer les écarts dans la distance de dispersion entre des populations dont les taux de femelles sont différents.

Les mesures que nous avons réalisées ont été effectuées dans des conditions qui correspondent aux stades « jeunes " des populations de thym. En effet, dans nos expérimentations, la densité est faible pour la migration du pollen $\left(4,7\right.$ individus $\left./ \mathrm{m}^{2}\right)$ et elle peut être considérée comme presque nulle pour celle des graines puisqu'aucun obstacle ne gêne la dissémination des diaspores lors des expérimentations. L'hétérogénéité topographique de la garrigue et le groupement des individus dans des populations à fortes densités constituent de nombreuses barrières à la dissémination (LEVIN \& KERSTER, 
1969). Ainsi, les valeurs obtenues peuvent correspondre à des cas de dissémination en milieu ouvert, comme il s'en forme après une perturbation.

Si les distances moyennes de dispersion des fruits sous l'action du vent sont relativement faibles, une certaine part peut être attribuée aux limitations du dispositif expérimental : le ventilateur émet un vent de force constante et égale à $5 \mathrm{~m}$ par seconde ; or, dans la garrigue, des vitesses supérieures peuvent être fréquemment observées.

D'une façon générale, la majorité des graines migrent très peu (de l'ordre du demi-mètre). Les travaux précédents (GouYon et al., 1987) invoquent dans leurs interprétations une faible migration des graines de thym ; nos résultats expérimentaux sont en accord avec cette hypothèse. Cependant, nos expérimentations mettent en évidence la possibilité de transfert de gènes sur de relativement longues distances (de l'ordre de quelques mètres), à la fois par le pollen et par les semences. De plus, les autres facteurs de dispersion qui n'ont pas été étudiés ici (hydrochorie et zoochorie notamment) peuvent certainement agir sur des distances beaucoup plus importantes (plusieurs kilomètres). Ces transferts étant fortement minoritaires, ils sont souvent négligés dans les formalisations mathématiques, alors qu'ils sont malgré tout primordiaux lors de la recolonisation des milieux perturbés. Comment expliquer autrement l'existence de thym dans des cultures récemment abandonnées et sur les points culminants de notre région?

\section{Voisinage d'une espèce gynodiö̈que}

Les estimations de $\mathrm{Ne}$ (tableau 3) couvrent d'un bout à l'autre la gamme de valeurs relevées dans la littérature pour d'autres végétaux (KERSTER \& LEVIN, 1968 ; LEVIN \& Kerster, 1968, 1969 ; SchaAl \& Levin, 1978 ; Beattie \& Culver, 1979 ; SchaAl, 1980 ; SCHMTt, 1980 ; CaHAlan \& Gliddon, 1985). Les fortes différences dans les valeurs de $\mathrm{Ne}$ sont attribuables aux variations de la densité. Les populations anciennes (Les Chênes et Le Vigan), du fait des faibles effectifs de leurs voisinages, sont nécessairement hétérogènes (par des effets de dérive), malgré leur âge, à la fois pour les génomes nucléaires et cytoplasmiques. Au contraire, dans la jeune population (station LJ2), la forte densité due à une colonisation en taches privilégie l'effet de fondation dû aux colonisateurs originels. Dans ce cas, les voisinages sont historiquement hétérogènes, mais la forte taille de chaque voisinage atténuera les effets de la dérive génétique (Belhassen \& Gouyon, 1987).

Il est indispensable de rappeler les critiques apportées aux modèles d'isolement par la distance. En effet, Felsenstein (1975) a montré que le modèle de MALÉcot (1948) repose sur des hypothèses incompatibles entre elles. En particulier, l'hypothèse d'une répartition uniforme des individus est incompatible avec l'hypothèse d'un tirage aléatoire du nombre de descendants dans une loi de Poisson de moyenne égale à 1 et celle d'une dispersion non uniforme des descendants. Il en serait de même pour le modèle de WrIGHT $(1943,1946)$, qui utiliserait plus indirectement les mêmes hypothèses (FELSENSTEIN, 1976). En fait, le modèle d'isolement par la distance doit être considéré comme une approximation du «stepping-stone model " (mais ici avec une distribution continue des individus) puisque les résultats de ces 2 modèles cö̈ncident d'une façon satisfaisante (FELSENSTEIN, 1976).

En l'absence d'une théorie générale, nous avons essayé de connaître le sens de variation de $\mathrm{Ne}$, en fonction des paramètres du modèle. Dans le cas des espèces 
gynodioïques, les valeurs des effectifs de voisinage varient fortement et sont influencées par les paramètres $t$ et $h$ (tableau 3 ) :

- $\mathrm{h}$ est négativement corrélé à $\mathrm{Ne}:$ quand $\mathrm{h}$ diminue, la proportion de femelles augmente et donc la variance de dispersion globale augmente puisque les femelles disséminent plus loin leurs gènes ;

$-\mathrm{h}$ est aussi négativement corrélé à $\mathrm{d}:$ quand une population vieillit, la proportion d'hermaphrodites augmente et la densité décroît (DOMMEE \& JACQUARD, 1985) ;

- l'influence de $\mathrm{h}$ et de $\mathrm{t}$ (et de leur interaction) sur $\mathrm{Ne}$ est très difficilement appréciable dans le cas d'espèces gynodioïques. En effet, Ne diminue lorsque le taux d'allofécondation est faible, car les gènes sont alors transmis deux à deux au lieu de l'être individuellement. D'autre part, un taux élevé de femelles (h faible) entraîne à la fois l'augmentation de la surface du voisinage et celle du taux d'allofécondation dans la population. Ceci aura donc pour effet un accroissement de $\mathrm{Ne}$. Cependant, si le taux de femelles est très élevé, le nombre d'hermaphrodites sera petit, ce qui engendrera une forte consanguinité dans la descendance et, par conséquent, $\mathrm{Ne}$ sera faible.

D'une façon générale, une population à fort taux de femelles sera composée de voisinages dont les surfaces et les effectifs seront plus grands. Le calcul du voisinage fournit ici une mesure des possibilités de structuration des populations naturelles en fonction du taux de femelles. En effet, WRIGHT prévoit l'apparition d'une différenciation génétique quand l'effectif génétique est inférieur à 200 individus (WRIGHT, 1946). Lorsque les populations de thym sont jeunes et donc riches en femelles (DOMMÉE \& JACQUARD, 1985 ; Couvet et al., 1986), Ne est supérieur à 200 (La Jasse 2, tableau 3). Par contre, dans les populations plus vieilles (Les Chênes, Le Vigan), $\mathrm{Ne}$ est de l'ordre de la dizaine d'individus. Ainsi, les populations vieilles à faible taux de femelles seront plus structurées spatialement que les populations plus jeunes.

\section{Conclusion}

La mesure des distances de migration des graines et du pollen chez Thymus vulgaris montre que cette plante disperse la majorité de ses gènes sur de très faibles distances. L'évaluation de la taille et de l'effectif du voisinage pour cette espèce gynodioïque nous a permis de mettre en évidence l'influence du taux de femelles sur les dimensions du voisinage : il apparaît, sur les bases de nos résultats expérimentaux, que l'augmentation du taux de femelles entraîne une augmentation de la taille et de l'effectif du voisinage. La connaissance de cette relation est fondamentale, puisque c'est à l'échelle du voisinage que se produisent les différenciations nucléocytoplasmiques qui conduisent à de fortes proportions de femelles (BelHassen et al., 1987 ; Couvet et al., 1986). Ainsi, la structuration spatiale des populations ne peut plus être négligée, et la notion de voisinage doit être intégrée aux outils d'analyse du maintien de la gynodioecie.

Cependant, il ne faut pas perdre de vue que toute mesure du voisinage est toujours effectuée à partir de valeurs moyennes de la variance de dispersion, du taux d'allofécondation, de la densité, et ne peut aboutir qu'à la définition d'un voisinage moyen. Les 10 p. 100 de fruits migrant 2 fois plus loin que la moyenne (fig. 2) et les transferts de pollen sur des distances égales à $9 \mathrm{~m}$, sont quantitativement dilués dans le flux génique dominant. Toute interprétation doit tenir compte de cette fraction non négligeable de pollen et de semences dispersés au-delà du voisinage, mais qui contribue aux 
échanges géniques intra-population et inter-population, permettant ainsi l'existence de la métapopulation (ensemble de populations qui se fondent les unes à partir des autres, Gill, 1978 ; Couvet et al., 1985a).

Reçu le 13 janvier 1986.

Accepté le 23 janvier 1987.

\section{Remerciements}

Nous remercions Mlles B. Bastrenta et D. Pomente, MM. Couvet, KJellberg et Valdeyron pour toute l'aide qu'ils ont bien voulu nous apporter.

\section{Références bibliographiques}

Barrowclough G.F., 1980. Gene flow, effective population sizes and genetic variance components in birds. Evolution, 34, 789-798.

Beattie A.J., Culver D.C., 1979. Neighborhood size in Viola. Evolution, 33, 1226-1229.

Begon M., Krimbas C.B., Loukas M., 1980. The genetics of Drosophila subobscura populations. XV. Effective size of a natural population estimated by three independent methods. Heredity, 45, 335-350.

Belhassen E., Gouyon P.H., 1987. Flux géniques, perturbations et gynodioecie chez Thymus vulgaris. Colloque National CNRS «Biologie des Populations", Lyon, 4-6 sept. 1986. 134-140. Université Claude Bernard. Lyon I.

Belhassen E., Pomente D., Trabaud L., Gouyon P.H., 1987. Recolonisation après incendie chez Thymus vulgaris L. : résistance des graines aux températures élevées. Acta OecologicalOecol. Plant., 8, 38-45.

BONNEMAISON., 1980. Etude stationnelle de la dynamique du maintien d'un polymorphisme génétique : cas de quatre populations naturelles de Thymus vulgaris L. Thèse de $3^{\mathrm{e}}$ cycle, U.S.T.L., Montpellier.

Brabant P., Gouyon P.H., Kjellberg F., Valdeyron G., Vernet P., 1980. Pollinisation studies in Thymus vulgaris L. (Labietae). Acta Oecol. Oecol. Plant., 1, 37-45.

Cahalan G.M., Gliddon C., 1985. Genetic neighbourhood size in Primula vulgaris. Heredity, 54, 64-70.

Couvet D., Gouyon P.H., KJellberg F., Olivieri I., Pomente D., Valdeyron G., 1985a. De-la métapopulation au voisinage : la génétique des populations en déséquilibre. Génét. Sél. Evol., 17, 407-414.

Couvet D., Gouyon P.H., Kjellberg F., Valdeyron G., 1985b. Génétique. La différenciation nucléocytoplasmique entre populations : une cause de l'existence de mâle-stériles dans les populations naturelles de thym. C. R. Acad. Sci., Paris, t 300, série III, no 18, 665-668.

Couvet D., Bonnemaison F., Gouyon P.H., 1986. The maintenance of females among hermaphrodites: the importance of nuclear-cytoplasmic interactions. Heredity, 57, 325-330.

CRAWFORD T.J., 1984a. The estimation of neighbourhood parameters for plant populations. Heredity, 52, 273-283.

Crawford T.J., 1984b. What is a population? In : SHorrocks B. (ed.), Evolutionary Ecology, 135-173, Blackwell Scientific Publications, Oxford.

Crow J.F., Kimura M., 1970. An Introduction to Population Genetics Theory. 591pp., Harper and Row publishers, New York.

Dommee B., Jacouard P., 1985. Gynodioecy in thyme, Thymus vulgaris L. : evidence from successionnal populations, In : Jacouard P., Heim G., Antonovics J.(ed.), Genetic Differentiation and Dispersal in Plants, 141-164, Springer-Verlag, Berlin. 
Felsenstein J., 1975. A pain in the torus : some difficulties with models of isolation by distance. Am. Nat., 109, 359-368.

FELSENSTEIn J., 1976. The theoretical population genetics of variable selection and migration. Ann. Rev. Genet., 10, 253-280.

Gill D.E., 1978. The metapopulation ecology of the red-spotted newt, Notophtalmus vitidescens (Rafinesque). Ecol. monogr., 48, 145-166.

Gliddon C., Belhassen E., Gouyon P.H., 1987. Genetic neighbourhoods in plants with diverse systems of mating and different patterns of growth. Heredity (sous presse).

Gouyon P.H., King E.B., Bonnet J.M., Valdeyron G., Vernet Ph., 1987. Seed migration and structure of plant populations. Oecologia (Berlin), 72, 92-94.

GrEENWOOD J.J.D., 1975. Effective population numbers in the snail Cepaea nemoralis. Evolution, 28, 513-526.

KerSTER H.W., 1964. Neighborhood size in the rusty lizard, Sceloporus olivaceus. Evolution, 18, 445-457.

Kerster H.W., LeVin D.A., 1968. Neighborhood size in Lithospermum caroliniense. Genetics, 60, 577-587.

LeVin D.A., Kerster H.W., 1968. Local gene dispersal in Phlox. Evolution, 22, 130-139.

LeVin D.A., Kerster H.W., 1969. Density-dependent gene dispersal in Liathris. Am. Nat., 103, 61-74.

Levin D.A., Kerster H.W., Niedzlek M., 1971. Pollinator flight directionality and its effect on pollen flow. Evolution, 25, 113-118.

Malécot G., 1948. Les mathématiques de l'hérédité. 60 p., Masson, Paris.

Olivieri I., SwanN M., Gouyon P.H., 1983. Reproduction system and colonizing strategy of two species of Cardus (Compositae). Oecologia (Berl.), 60, 114-117.

SchaAl B.A., 1980. Measurement of gene flow in Lupinus texensis. Nature, 284, 450-451.

SChaAl B.A., LeVIN D.A, 1978. Morphological differentiation and neighborhood size in Liathris cylindracea. Am. J. Bot., 65, 923-928.

Schmirt J., 1980. Pollinator foraging behavior and gene dispersal in Senecio (Compositae). Evolution, 34, 934-943.

STOCKHOUSE R.E., 1976. A new method for studing pollen dispersal using micronized fluorescent dusts. Am. Midl. Nat., 96, 241-245.

Thomson J.D., Plowright R.C., 1980. Pollen carryover, nectar rewards, and pollinator behavior, with special reference to Diervilla lonicera. Oecologia, 46, 68-74.

ValdeYron G., Dommee B., Vernet P., 1977. Self-fertilisation in male fertile plants of a gynodioecious species: Thymus vulgaris L. Heredity, 39, 243-249.

WASER N.M., Price M.V., 1982. A comparison of pollen and fluorescent dye carryover by natural pollinator of Ipomopsis aggregata (Polemoniaceae). Ecology, 63, 1168-1172.

Wright S., 1931. Evolution in Mendelian populations. Genetics, 16, 97-159.

WRIGHT S., 1943. Isolation by distance. Genetics, 28, 114-138.

WRIGHT S., 1946. Isolation by distance under diverse systems of mating. Genetics, 31, 39-59. 